\title{
Real-time observation of $\mathrm{Cu}_{2} \mathrm{ZnSn}(\mathrm{S}, \mathrm{Se})_{4}$ solar cell absorber layer formation from nanoparticle precursors ${ }^{\dagger}$
}

\author{
Roland Mainz, ${ }^{* a}$ Bryce Walker, ${ }^{b}$ Sebastian S. Schmidt,${ }^{a}$ Ole Zander, ${ }^{a}$ Alfons Weber, ${ }^{a}$ Humberto \\ Rodriguez-Alvarez, ${ }^{a \ddagger}$ Justus Just, ${ }^{a}$ Manuela Klaus, ${ }^{a}$ Rakesh Agrawal, ${ }^{b}$ Thomas Unold ${ }^{a}$ \\ ${ }^{a}$ Helmholtz-Zentrum Berlin für Materialien und Energie, Hahn-Meitner-Platz 1, 14109 Berlin, Germany. \\ ${ }^{b}$ School of Chemical Engineering, Purdue University, West Lafayette, IN 47906, USA. \\ *E-mail: roland.mainz@helmholtz-berlin.de
}

\begin{abstract}
The selenization of $\mathrm{Cu}-\mathrm{Zn}$-Sn-S nanocrystals is a promising route for the fabrication of low-cost thin film solar cells. However, the reaction pathway of this process is not completely understood. Here, the evolution of phase formation, grain size, and elemental distributions is investigated during the selenization of $\mathrm{Cu}-\mathrm{Zn}-\mathrm{Sn}-\mathrm{S}$ nanoparticle precursor thin films by synchrotron-based in situ energy-dispersive X-ray diffraction and fluorescence analysis as well as by ex situ electron microscopy. The precursor films are heated in a closed volume inside a vacuum chamber under presence of selenium vapor while diffraction and fluorescence signals are recorded. The presented results reveal that during the selenization the cations diffuse to the surface to form large grains on top of the nanoparticle layer and the selenization of the film takes place in two simultaneous reactions: 1) a direct and fast formation of large grained selenides, starting with copper selenide which is subsequently transformed into $\mathrm{Cu}_{2} \mathrm{ZnSnSe}_{4}$; 2) a slower selenization of the remaining nanoparticles. As a consequence of the initial formation of copper selenides at the surface, the subsequent formation of CZTSe starts under $\mathrm{Cu}$-rich conditions despite an overall Cu-poor composition of the film. The implications of this process path on the film quality is discussed. Additionally, the proposed growth model provides an explanation of the previously observed accumulation of carbon from the nanoparticle precursor beneath the large grained layer.
\end{abstract}

\section{Introduction}

Absorber films of low-cost and high-efficiency solar cells have to fulfill two seemingly conflicting requirements: First, the absorber has to be of excellent semiconducting quality to avoid losses by electron-hole recombination. Second, the deposition process should be fast and require a minimum in energy consumption and technical equipment.

Compound semiconductor materials such as copper indium gallium diselenide and copper zinc tin sulfide can be deposited as nanoparticles dispersed in a solvent by fast and easily scalable processes resulting in homogenous and smooth films in order to provide low-cost deposition. ${ }^{1-6}$ It has been demonstrated that $\mathrm{Cu}-\mathrm{Zn}-\mathrm{Sn}-\mathrm{S}$ nanoparticle films can be transferred into microcrystalline kesterite $\mathrm{Cu}_{2} \mathrm{ZnSn}(\mathrm{S}, \mathrm{Se})_{4}$ (CZTSSe) absorber films for solar cells with energy conversion efficiencies of up to $7.2 \%$ by selenization at $500{ }^{\circ} \mathrm{C} .{ }^{2}$ A partial substitution of $\mathrm{Sn}$ by Ge lead to a further increased efficiency of $8.4 \%{ }^{7}$ To be able to easily influence the composition of the CZTSSe film, Cao et al. have recently used mixtures of binary and ternary nanoparticles in a similar process and reached so- lar cell efficiencies of up to $8.5 \% .{ }^{8}$ Even though the efficiencies reached with these approaches are still behind the highest efficiencies reached with CZTSSe $\left(11.1 \%^{9}\right)$, a synthesis via the nanoparticle route is attractive for low-cost solar module production. A main advantage of the deposition of nanoparticle inks over other deposition processes used for the synthesis of CZTSSe films - such as sputtering, ${ }^{10,11}$ thermal evaporation, ${ }^{12-15}$ or pulsed laser deposition ${ }^{16}$ - is that neither a vacuum is needed for the deposition of the precursor film, nor does it rely on toxic solvents.

Even though the formation of solar cell absorber films from nanoparticles has attracted a lot of attention and despite the remarkable success of this approach, the reaction path during the transition from the nanoparticles to large grains is not completely clarified. Yet, a detailed understanding of the mechanisms taking place during the formation of large grains out of nanoparticles is essential for further improvement of the processes. Guo et al. suggested that the formation of large grains is enabled by a lattice expansion due to the replacement of sulfur by selenium, leading to a compaction of the film and thereby causing the nanoparticles to merge. ${ }^{1}$ However, it re- 
mains unclear, how the formation of large grains is correlated with the selenization of the nanoparticles, whether intermediate phases form during the transition and why carbon from the precursor film is left underneath the large grained layer. ${ }^{7,8}$

This work investigates these issues by studying the grain growth, phase formation, and elemental distributions during the selenization of $\mathrm{Cu}-\mathrm{Zn}-\mathrm{Sn}-\mathrm{S}$ precursor films by synchrotron based in situ energy-dispersive X-ray diffraction and fluorescence (EDXRD/XRF) in combination with ex situ crosssectional electron microscope analysis. The time-resolved data presented here reveal insights into the process that cannot be accessed by ex situ methods alone. We find that large copper selenide grains are initially formed at the surface which are subsequently transformed into large $\mathrm{Cu}_{2} \mathrm{ZnSn}\left(\mathrm{S}, \mathrm{Se}_{4}\right)$ grains with a low or vanishing sulfur content. Our interpretation of the results points out possible strategies for a deliberate manipulation of the reaction path. This will help to further improve this process as well as develop new process routes.

\section{Experimental Section}

$\mathrm{Cu}-\mathrm{Zn}-\mathrm{Sn}-\mathrm{S}$ nanoparticles were formed as reported previously $^{2,17}$ with a $\mathrm{Cu}: \mathrm{Zn}: \mathrm{Sn}$ atomic ratio of 1.76:1.05:1. The particles were suspended in hexanethiol and deposited onto molybdenum coated soda-lime glass substrates by doctor blading (see ESI $\dagger$ for details on materials and synthesis).

These samples were then annealed in selenium vapor inside a cylindrical graphite reaction box placed inside a vacuum chamber. The annealing chamber ${ }^{18}$ was coupled to the polychromatic EDDI beam line ${ }^{19}$ of the BESSY II synchrotron facility. During the processes, diffraction and fluorescence signals were recorded in an energy-dispersive high-purity $\mathrm{Ge}$ detector under a scattering angle of $2 \vartheta=6.248 \pm 0.002^{\circ}$. The relationship between lattice plane spacing $d_{\mathrm{hkl}}$ of a crystalline phase and the photon energy $E_{\mathrm{hkl}}$ of the corresponding diffraction line follows the energy-dispersive Bragg law $E_{\mathrm{hkl}}=h c /\left(2 d_{\mathrm{hkl}} \sin \vartheta\right)$, where $h$ is Planck's constant and $c$ the speed of light. Complete spectra were recorded every $10 \mathrm{sec}-$ onds.

For the selenization processes, $160 \mathrm{mg}$ elemental Se was placed in a small ceramic container inside the reaction box next to the sample. The box was sealed from the vacuum of the chamber by a valve after a base pressure of $\sim 10^{-4}$ mbar was reached. The samples, the reaction box and the selenium were heated simultaneously. (More experimental details can be found in $\mathrm{ESI} \dagger$.)

\section{Results and Discussion}

To reveal and understand some of the finer intricacies and potential transformations that may be present during the sinter- ing step, in situ studies with slow heating $\left(0.05 \mathrm{~K} \mathrm{~s}^{-1}\right)$ are presented and discussed first. Subsequently, the influence of the heating rate on the reaction path is studied by comparison of the results from the slow heating process to results of a selenization by fast heating $\left(1.7 \mathrm{~K} \mathrm{~s}^{-1}\right)$. In previous studies, best solar cell results were reached with selenization by a fast heating of the samples to $500^{\circ} \mathrm{C}$ with a total annealing time of 20 minutes. $^{2}$

\subsection{Selenization by slow heating}

In situ EDXRD/XRF spectra recorded during the slow selenization process of a $\mathrm{Cu}-\mathrm{Zn}-\mathrm{Sn}-\mathrm{S}$ nanoparticle film with a heating rate of $0.05 \mathrm{~K} \mathrm{~s}^{-1}$ are presented in Fig. 1. The graph shows color-coded intensities of diffracted photons as function of photon energy and process time. The top part of the graph shows the temperature measured by the thermocouple placed inside the reaction box (see ESI $\dagger$ for details). At the beginning of the process $(t=0)$, the color-coded diffraction signals show a broad signal $(\Sigma 1)$ at the expected position of the (112) reflex of tetragonal $\mathrm{Cu}_{2} \mathrm{ZnSnS}_{4}$ (CZTS). At the end of the heating ramp ( $t=130 \mathrm{~min}$.) this signal has disappeared and several sharp signals at the expected positions of tetragonal $\mathrm{Cu}_{2} \mathrm{ZnSnSe}_{4}$ (CZTSe) are visible. We note that the main reflexes of CZTSe (112), (204), and (116/312) coincide with reflexes of cubic $\mathrm{ZnSe}^{20}$ and cubic ${ }^{21}$ or monoclinic ${ }^{22}$ $\mathrm{Cu}_{2} \mathrm{SnSe}_{3}$. Because of this ambiguity we denote the signal at the position of CZTSe (112) by $\Sigma 2$. In contrast, the weak reflexes at the positions of CZTSe (101), (202), and (211) cannot stem from the cubic or monoclinic phases and reveal the formation of tetragonal CZTSe.

In addition to the diffraction signals, Se fluorescence signals arise during the process, showing the incorporation of $\mathrm{Se}$ into the film (Fig. 1). Sn fluorescence signals are present throughout the process. The intensity of $\mathrm{Sn}-\mathrm{K} \alpha$ decreases during incorporation of Se. At $500{ }^{\circ} \mathrm{C}$, when Se incorporation into the film is complete, $\mathrm{Sn}-\mathrm{K} \alpha$ has decreased by about $(8 \pm 1) \%$. The main share of this decrease can be explained by attenuation of the Sn fluorescence by the incorporated Se. From model calculations, ${ }^{23}$ a decrease of the $\mathrm{Sn}-\mathrm{K} \alpha$ intensity by $(6.5 \pm 1) \%$ is expected if sulfur in CZTS is replaced by selenium. From the comparison of the measured and the calculated intensities, we can conclude that at $500{ }^{\circ} \mathrm{C}$, the loss of $\mathrm{Sn}$ can be limited to a maximum of $4 \%$. At $550{ }^{\circ} \mathrm{C}$, the $\mathrm{Sn}-\mathrm{K} \alpha$ intensity has decreased by $(14 \pm 1) \%$, revealing significant $\mathrm{Sn}$ loss during the last stage of the heating process. Loss of Sn from kesterite at high temperatures is a phenomenon known from the literature. ${ }^{14,24,25}$

Both, a shift of the initial signal $\Sigma 1$ from the position of CZTS to the position of CZTSe and a sharpening of the signal is expected if (1) sulfur in CZTS is replaced by selenium - resulting in an increase of lattice constants - and (2) large 


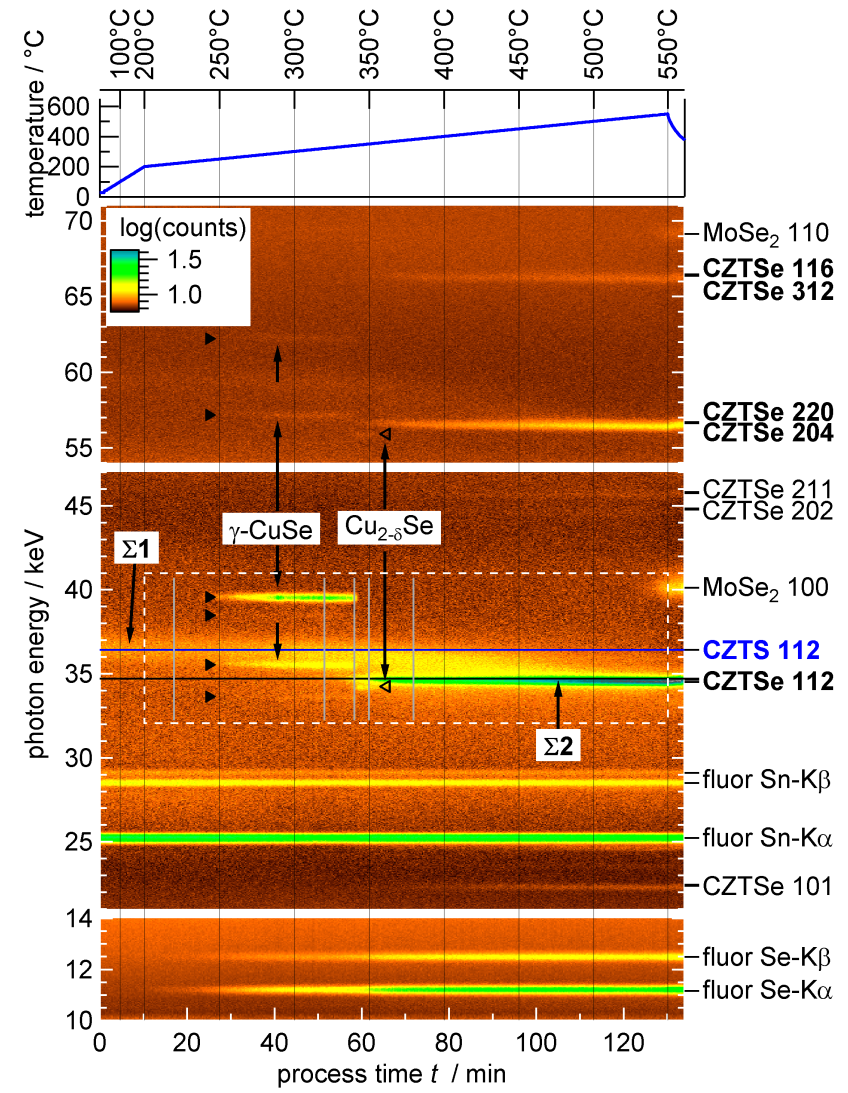

Figure 1 Color-coded presentation of the EDXRD/XRF intensities as function of photon energy and process time recorded during the selenization of a $\mathrm{Cu}-\mathrm{Zn}-\mathrm{Sn}-\mathrm{S}$ nanoparticle film with a heating rate of $0.05 \mathrm{~K} \mathrm{~s}^{-1}$ (preceded by a faster heating ramp to 200C). On the right hand side the energy positions of reference fluorescence lines (fluor) and of reference diffraction lines calculated from data of the ICDD data base are marked (CZTS: card 026-0575; CZTSe: 070-8930; MoSe2: 029-0914). The filled triangles mark the positions of $\mathrm{CuSe}$ (086-1240) and the empty triangles mark the positions of $\mathrm{Cu}_{2-\delta} \mathrm{Se}$ (073-2712). The gray vertical lines mark ranges of single spectra that are presented in Fig. 2. The white dashed line marks the region shown enlarged in Fig. 3.

grains are formed during the process - resulting in a decrease of the width of the diffraction peaks. Indeed, a shift of the broad signal $\Sigma 1$ from the CZTS (112) to the CZTSe (112) position can be seen in Fig. 1. However, the formation of the sharp signal $\Sigma 2$ at the position of CZTSe (112) is not a result of a shift and sharpening of the broad $\Sigma 1$ peak. Instead, at a temperature of about $350{ }^{\circ} \mathrm{C}$ this signal directly appears with a narrow peak width and at its final photon energy position expected for CZTSe (112). This shows that there is no continuous transition from the sulfide nanoparticles to large CZTSe grains and therefore contradicts the model of grain growth by selenization and merging of the nanoparticles.
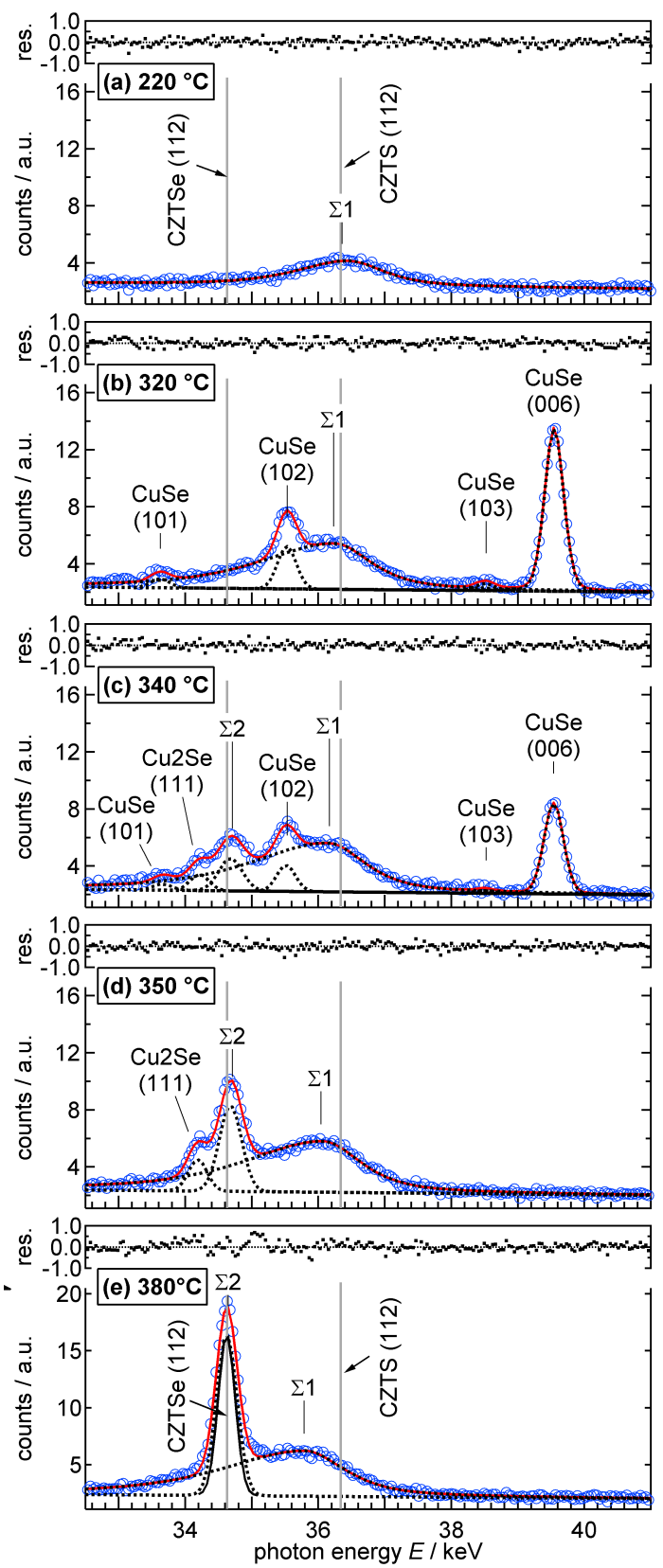

Figure 2 Single EDXRD spectra (circles) in the energy range from 32.5 to $41 \mathrm{keV}$ recorded at (a) $220{ }^{\circ} \mathrm{C}$, (b) $320{ }^{\circ} \mathrm{C}$, (c) $340{ }^{\circ} \mathrm{C}$, (d) $350{ }^{\circ} \mathrm{C}$, and (e) $380{ }^{\circ} \mathrm{C}$ during the slow selenization presented in Fig. 1. Additionally, the graphs show the result of multi-peak fits (single peaks: black dotted lines, sum of peaks: red solid line). The peak drawn by a black solid in (e) represents the energy resolution of the detector with a full width at half maximum of $0.3 \mathrm{keV}$. The vertical grey lines mark the reference positions for the reflexes CZTS (112) (ICDD card 026-0575) and CZTSe (112) (ICDD card 070-8930). 


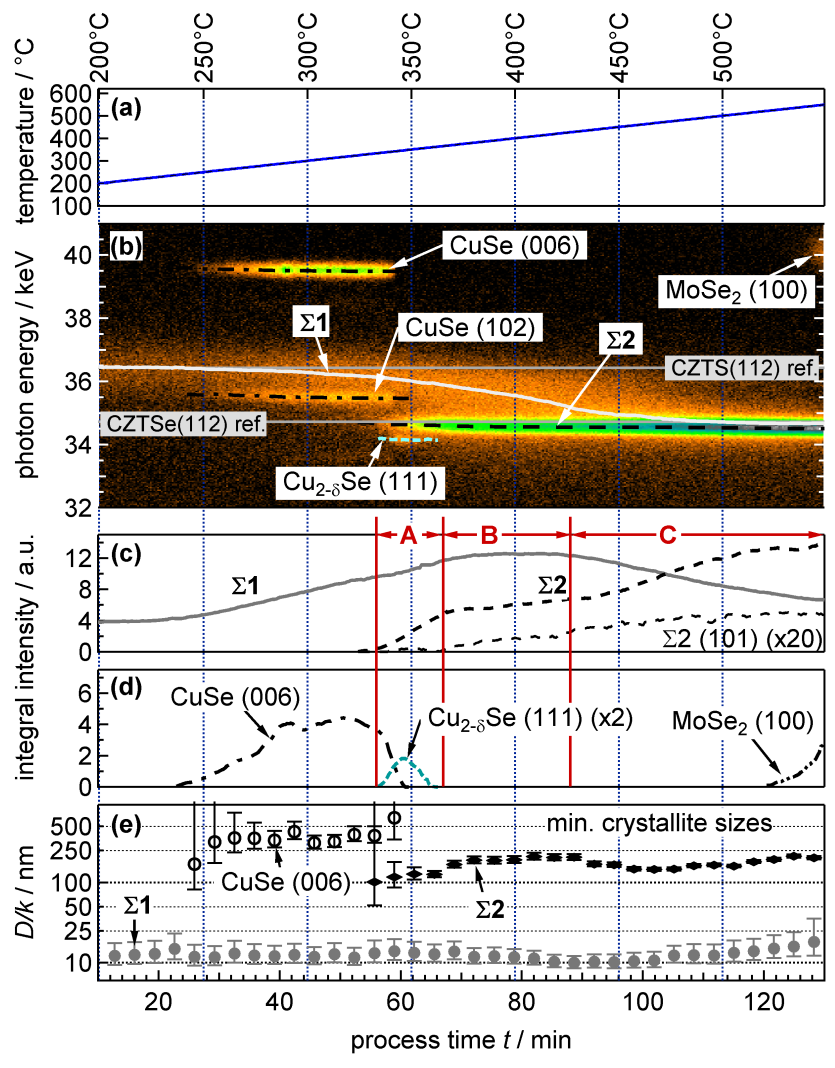

Figure 3 Time-resolved EDXRD data and peak properties of the selenization of a $\mathrm{Cu}-\mathrm{Zn}-\mathrm{Sn}-\mathrm{S}$ nanoparticle film with a slow heating rate of $0.05 \mathrm{~K} \mathrm{~s}^{-1}$. a) Temperature measured by the thermocouple placed inside the reaction box. b) Color-coded signal intensities and position of peak maxima versus photon energy and process time. c) and d) Integral intensities of the diffraction signals determined by multi-peak fitting. e) Minimum crystallite sizes $D$ divided by the Scherrer constant $k$, which is close to one for most crystallite shapes. ${ }^{26}$ The error bars present double standard deviations calculated from the uncertainties resulting from the Levenberg-Marquardt fit algorithm.

An additional important feature of the process revealed by Fig. 1 is the appearance of intermediate, sharp signals between $240{ }^{\circ} \mathrm{C}$ and $340{ }^{\circ} \mathrm{C}$ which can be attributed to hexagonal $\mathrm{CuSe}$. The filled triangles in Fig. 1 mark reference positions for hexagonal $\mathrm{CuSe}$ calculated from literature data, ${ }^{27}$ showing a good agreement with the positions of the signals. A lattice parameter fit, matching calculated reflex positions to the measured positions, confirmed a hexagonal structure. (For more details on the analysis of the lattice parameters of $\mathrm{CuSe}$, see ESI†.)

When the CuSe signals disappear at $340{ }^{\circ} \mathrm{C}$, two weak signals, which can be attributed to $\mathrm{Cu}_{2-\delta} \mathrm{Se}$, appear simultaneously with the signals at the positions of CZTSe. The empty triangles mark reference positions of $\mathrm{Cu}_{2-\delta} \mathrm{Se}$ reflexes. ${ }^{28}$
They disappear at $360{ }^{\circ} \mathrm{C}$. The attribution of these weak reflexes to $\mathrm{Cu}_{2-\delta} \mathrm{Se}$ is supported by additional investigations wherein we repeated the presented process, but started to cool down immediately after the $\mathrm{Cu}_{2-\delta}$ Se reflexes occurred. This resulted in an disappearance of these reflexes and recurrence of the $\mathrm{CuSe}$ reflexes, which is expected from the $\mathrm{Cu}-\mathrm{Se}$ phase diagram under Se-rich conditions ${ }^{29}$.

\subsubsection{Formation of large grains by cation diffusion.}

The peak widths of the signals provide information about crystallite sizes (which is the size of a domain of coherent scattering) and therefore allow important insights into the transition of the nanoparticles to large grains. In the time and energy resolved representation of the EDXRD data in Fig. 1 it is difficult to identify the partially overlapping diffraction reflexes with low intensities as well as the shapes of the signals. They can, however, be identified by the analysis of single spectra, as shown in Fig. 2. (The points in process time at which these spectra were recorded are marked in Fig. 1 by vertical lines.) Compared to the broad signal $\Sigma 1$ (Fig. 2a-e), the signals of $\mathrm{CuSe}$ (b and c), $\mathrm{Cu}_{2-\delta} \mathrm{Se}$ (c and d), and CZTSe (d and e) are much sharper with a width close to the resolution of the detector. The detector resolution is visualized by the peak drawn by a black solid line in Fig. 2e with a full width at half maximum of $0.3 \mathrm{keV}$. As smaller crystallites exhibit wider peaks, this indicates that the grains that induce the sharp peaks are much larger than the initial nanoparticles, which induce the broad $\Sigma 1$ signal.

A precise determination of the crystallite sizes from the shape of the diffraction signals is complicated by the fact that the signal shape can be additionally broadened by microstrain $^{30}$ and composition gradients. ${ }^{31}$ A determination of crystallite sizes from the diffraction data remains ambiguous to a certain extent mainly due to the possible occurrence of $\mathrm{S} / \mathrm{Se}$ gradients. The asymmetric broadening of the $\Sigma 1$ signal during its shift towards the position of CZTSe can be explained by an inhomogeneous incorporation of Se into the nanoparticle layer (Fig. 2a-e). Despite this difficulty, a minimum crystallite size can be determined. The calculation of the minimum crystallite size for a given reflex follows the logic that if the crystallites were smaller than this value, the reflex would be wider - with or without the presence of micro strain or composition gradients. (A detailed description of the calculation of the minimum crystallite sizes is given in the $\mathrm{ESI} \dagger$.)

To extract the shape of the peaks which is needed for the calculation of the minimum crystallite sizes as well as for integral intensities and energy positions of the signals as functions of time, a multi-peak fit using pseudo-Voigt profiles was performed on each recorded spectrum. Resulting peak profiles and their deviations from the measured data (res.) were added to Fig. 2. To account for the asymmetric nature of the broad 

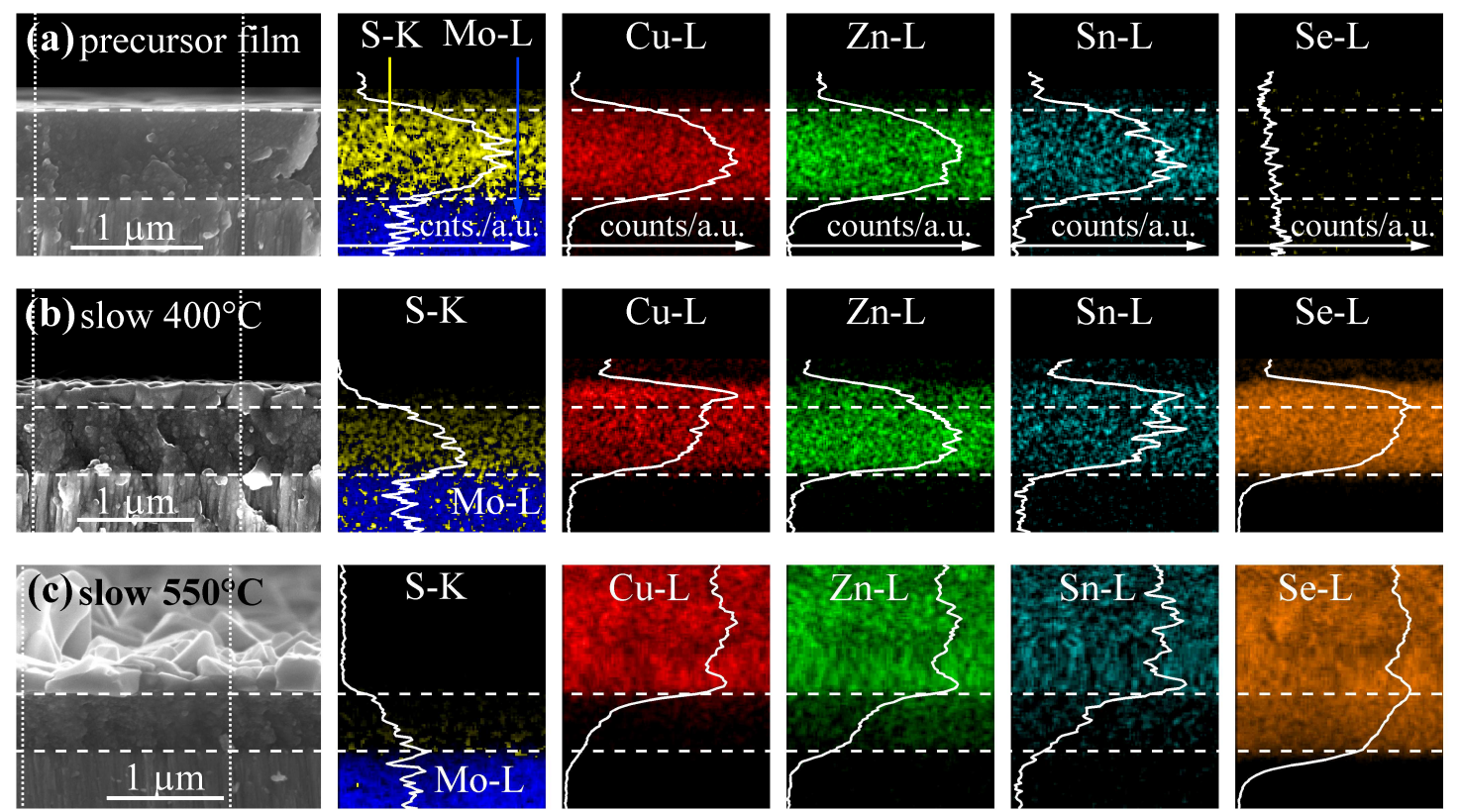

Figure 4 SEM images and EDS mappings of cross-sections of the $\mathrm{Cu}-\mathrm{Zn}-\mathrm{Sn}-\mathrm{S}$ nanoparticle precursor (a) and of the samples that were selenized by slow heating $\left(0.05 \mathrm{~K} \mathrm{~s}^{-1}\right)$ up to $400^{\circ} \mathrm{C}$ (b) and up to $550^{\circ} \mathrm{C}(\mathrm{c})$.

peak marked by $\Sigma 1$, the signal was fitted with an asymmetric pseudo-Voigt profile.

The time evolution of the peak properties resulting from the multi-peak fits and from the calculation of minimum crystallite sizes are shown in Fig. 3. Fig. 3b shows an enlarged presentation of the $\Sigma 1$ and $\Sigma 2$ region of the time-resolved EDXRD data from Fig. 1. The thick lines in Fig. 3b mark the photon energy peak positions of the maxima of $\Sigma 1, \Sigma 2$, CuSe, and $\mathrm{Cu}_{2-\delta} \mathrm{Se}$ as functions of process time. Integral signal intensities, which were calculated from the fit parameters, are plotted in Fig. 3c and d. Minimum crystallite sizes calculated from the signals $\mathrm{CuSe}(006), \Sigma 1$, and $\Sigma 2$ are plotted in $3 \mathrm{e}$. The minimum crystallite size for $\Sigma 1$ of 10 to $20 \mathrm{~nm}$ are consistent with the sizes observed by transmission electron microscopy (TEM). ${ }^{17}$ Its value stays nearly constant throughout the process.

Remarkably, immediately after CuSe started to form at 240 ${ }^{\circ} \mathrm{C}$ its minimum average crystallite size is larger than $100 \mathrm{~nm}$ and then increases above $200 \mathrm{~nm}$. Also, the sizes determined from $\Sigma 2$ are immediately larger than $100 \mathrm{~nm}$. (We note that the signals attributed to $\mathrm{Cu}_{2-\delta} \mathrm{Se}$ are too small to reliably determine minimum crystallite sizes for this phase.)

The formation of large $\mathrm{Cu}$-Se grains has an important consequence. Since the precursor film initially consists of $\mathrm{Cu}-$ $\mathrm{Zn}-\mathrm{Sn}-\mathrm{S}$ nanoparticles, the formation of large $\mathrm{Cu}-\mathrm{Se}$ grains is necessarily connected with a spatial separation of $\mathrm{Cu}$ from $\mathrm{Zn}$ and Sn. There are two principle possibilities for this separation to take place: 1) Sn and $\mathrm{Zn}$ move away from areas at which large $\mathrm{Cu}$-Se grains form. 2) Cu diffuses to a location where no $\mathrm{Sn}$ and $\mathrm{Zn}$ is present, that is to the surface of the sample. The second possibility is supported by cross-sectional scanning electron microscopy (SEM) images and elemental energy-dispersive X-ray spectroscopy (EDS) mappings shown in Fig. 4. The upper images (a) show a nanoparticle precursor film, which has not been annealed. Here, the distributions of $\mathrm{Cu}, \mathrm{Zn}$, and $\mathrm{Sn}$ correlate. The images in the middle (b) show a sample that was selenized with the slow heating process, which was interrupted at $400{ }^{\circ} \mathrm{C}$, after $\mathrm{Cu}$-Se disappeared. Here, the SEM image on the left shows a $200 \mathrm{~nm}$ thick layer of large grains at the surface of the film. Additionally, the EDS mappings reveal an increased $\mathrm{Cu}$ concentration, the presence of $\mathrm{Se}$ and the absence of $\mathrm{S}$ near the surface. This suggests that the large $\mathrm{Cu}-\mathrm{Se}$ grains seen between $240{ }^{\circ} \mathrm{C}$ and $360{ }^{\circ} \mathrm{C}$, are formed at the surface. Additionally, an increased $\mathrm{Zn} /(\mathrm{Cu}+\mathrm{Sn})$ atomic ration can be seen in the bottom layer. Measurements by X-ray absorption spectroscopy at the K-edge of sulfur (not shown) indicate that approximately $43 \%$ of the $\mathrm{S}$ left in the $400{ }^{\circ} \mathrm{C}$ sample is bound in $\mathrm{Zn}(\mathrm{S}, \mathrm{Se})$. (For details on the technique see e.g. Just et al. ${ }^{32}$ )

In the large grained layer of the film that was heated up to $550{ }^{\circ} \mathrm{C}$, the distributions of $\mathrm{Cu}, \mathrm{Zn}$, and $\mathrm{Sn}$ correlate again (Fig. 4c). These results reveal that cation diffusion takes place during the selenization of the nanoparticle film.

The SEM images in Fig. 4b and c show two distinct layers with a clear interface (horizontal dashed line) with a large grained layer at the top. The interface of the sample that was 
annealed up to $400{ }^{\circ} \mathrm{C}$ (Fig. 4b) was further analyzed by high-resolution transmission electron microscopy (HR-TEM) shown in Fig. 5a. The SEM inset shows an enlarged section of the SEM image in Fig. 4b. The upper part of the image shows a large area of uniform lattice planes, which therefore is attributed to a single grain (denoted by "Large grain"). The border of this area is marked by a white dash-dotted line. In contrast, the lower part shows several smaller areas of uniform lattice planes with diameters in the order of up to 10 $\mathrm{nm}$. The numbers give the lattice plane spacings for the corresponding areas extracted by Fourier transformations. They are close to the lattice plane spacings of CZTSe $112(3.28 \AA)$ and of CZTSe 204/220 (2.01 $\AA$ ). ${ }^{33}$ The deviations from these values - as well as from the corresponding lattice planes of $\mathrm{ZnSe}$ or CTSe - are within the resolution of the measurement. In contrast, they significantly deviate from the lattice plane spacings of CZTS 112 (3.13 $)$ and CZTS 204/220 (1.92 $).{ }^{34}$ The insets of Fig. 5a show transmission electron diffractograms (TED) recorded in the large grained and the nanoparticle layer, respectively. The innermost circle corresponds to the 112 and the second inner circles to the 204/220 lattice planes of CZTSe. In a TEM-EDS line scan across the interface of the large grain layer and the nanoparticle layer an approximately $50 \mathrm{~nm}$ thick Zn-rich layer at the bottom of the large grained layer can be observed (Fig. 5b). A TEM-EDS mapping from which the line scan was extracted shows a laterally uniform thickness of the ZnSe layer (see Fig. S3†). Therefore the part of the large grain seen in the graph should partially consist of $\mathrm{ZnSe}$.

The graphs in Fig. 5 show that nanoparticles with a high $\mathrm{Se} / \mathrm{S}$ ratio and of sizes in the order of $10 \mathrm{~nm}$ exist in the direct neighborhood of a large grain. Even though the shown area does not provide good statistics, this observation supports a formation of large grains on top of the nanoparticle layer instead of a continuous growth of nanoparticles.

\subsubsection{Formation of $\mathrm{Cu}_{2} \mathrm{ZnSnSe}_{4}$.}

The intensity of the $\Sigma 2$ signal at the energy position of CZTSe (112) shows three distinct time periods (denoted by A, B, and $\mathrm{C}$ in Fig. 3c):

- A: The $\Sigma 2$ intensity rises rapidly. No significant CZTSe (101) reflex is present.

- B: The $\Sigma 2$ intensity rises slowly. The CZTSe (101) reflex starts to rise

- C: An increasing rise of the $\Sigma 2$ intensity is accompanied by a decline in $\Sigma 1$ intensity.

Regarding period A we make two additional observations: (i) $\mathrm{All} \mathrm{Cu}$-Se intensities disappear during this period, (ii) intensities corresponding to the $\mathrm{Cu}_{2-\delta}$ Se peaks appear simultane-
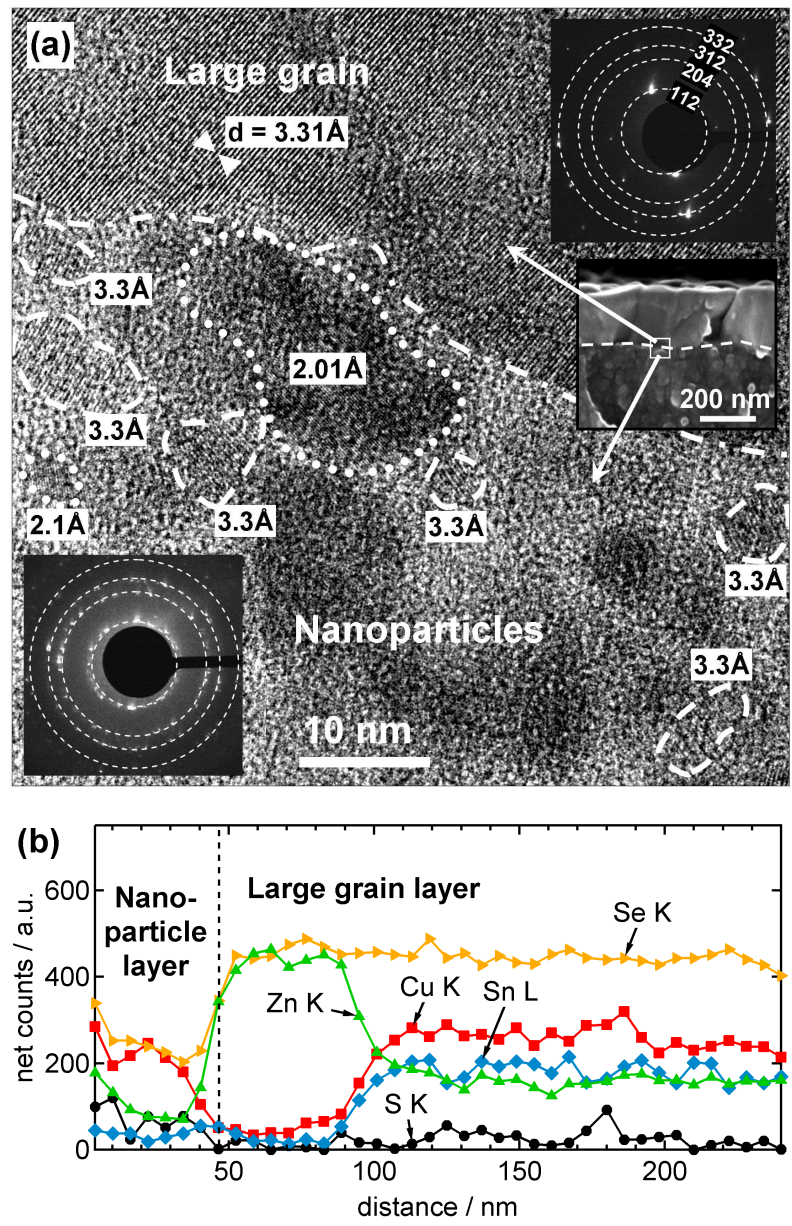

Figure 5 (a) HR-TEM image of a region around the interface of the large grain layer and the nanoparticle layer of the sample that was selenized by slow heating up to $400^{\circ} \mathrm{C}$. The two insets show electron diffraction patterns measured at the large grain area ([513] zone axis) and at the nanoparticles area, respectively. The dashed rings in both insets have equal diameters. The innermost circle corresponds to the CZTSe 112 planes and the second inner circle to the 204/220 planes. (b) TEM-EDX line scan across the interface of the nanoparticle layer and the large grained layer. The position of the zero point of the $\mathrm{x}$-axis is arbitrary.

ously with $\Sigma 2$ at the beginning of this period and then disappear by the end of this period, (iii) the weak CZTSe (101) signal does not show a significant intensity in this time period and only rises in period $\mathrm{B}$.

The disappearance of the $\mathrm{Cu}-\mathrm{Se}$ signals during the initial rapid rise of the $\Sigma 2$ signal implies that $\mathrm{Cu}-\mathrm{Se}$ is converted into the phase causing the $\Sigma 2$ signal. The absence of the CZTSe (101) indicates that in this period the signal $\Sigma 2$ is not caused by tetragonal CZTSe, but rather by a cubic phase. This leaves us with the following possibilities: (1) During this period the sintered CZTSe phase is oriented and therefore either does not 


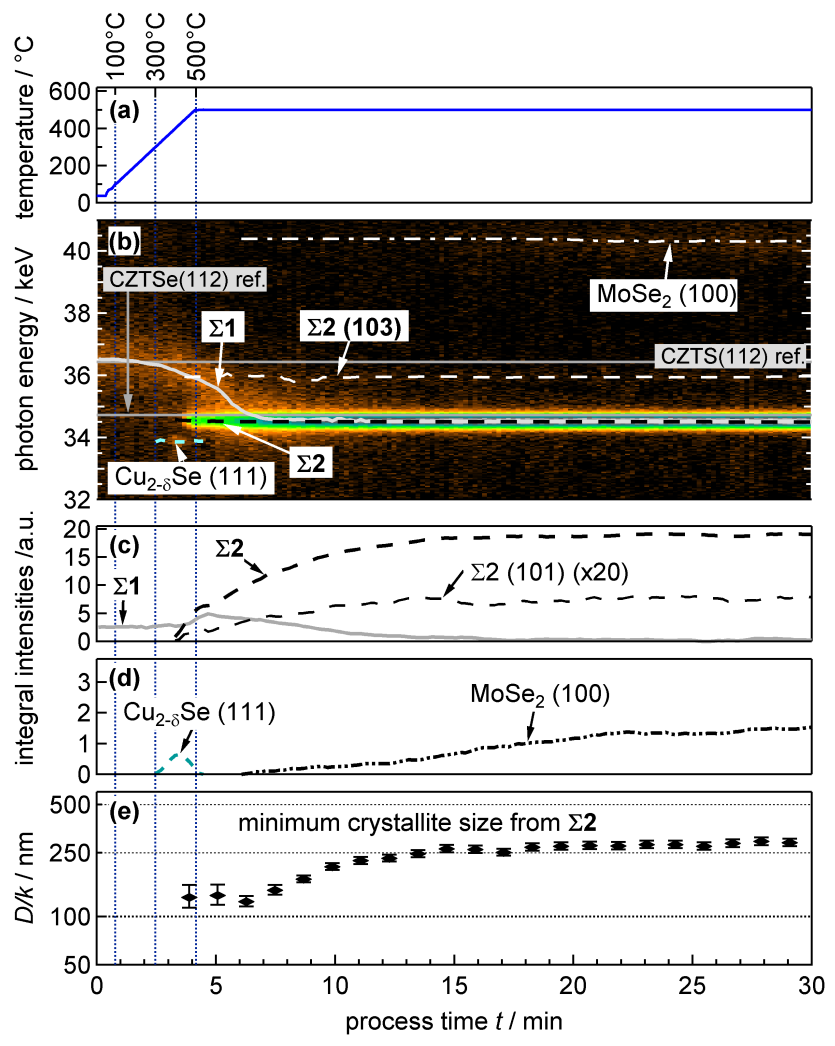

Figure 6 Time-resolved EDXRD data and peak properties of the selenization of a $\mathrm{Cu}-\mathrm{Zn}$-Sn-S nanoparticle film with a fast heating rate of $1.7 \mathrm{~K} \mathrm{~s}^{-1}$. Description similar to Fig. 3. The temperature of the film is estimated to lag up to $100{ }^{\circ} \mathrm{C}$ behind the measured temperature during the heating ramp (also see $\mathrm{ESI} \dagger$ ).

show a CZTSe (101) signal or this signal is only very weak. (2) Initially CZTSe forms as a cubic phase with a disordered occupation of the cation sites. (3) Cubic phases such as $\mathrm{ZnSe}$ or (4) $\mathrm{Cu}_{2} \mathrm{SnSe}_{3}$ (CTSe) are formed prior to CZTSe.

The EDS mappings of the sample for which the heating was interrupted at $400{ }^{\circ} \mathrm{C}$ (in period B) supports a CTSe formation at the surface since an enrichment of $\mathrm{Cu}$ and $\mathrm{Sn}$ in the large grained layer compared to $\mathrm{Zn}$ can be seen (Fig. 4b). Additionally, the TEM-EDS measurements on the same sample shown in Fig. 5 revealed that a thin layer of $\mathrm{ZnSe}$ exists at the bottom of the large grained layer. We conclude that in period A, mainly tin diffuses from the nanoparticle film into the copper selenide at the surface to form CTSe, followed by Zn which finally lead to the transformation of the large grains into CZTSe. The formation of tetragonal CZTSe is revealed by the rise of the (101) reflex (Fig. 3c) and by the correlation of the spatial distributions of $\mathrm{Cu}, \mathrm{Zn}$, and $\mathrm{Sn}$ in the final film (Fig. 4c).

Finally, in period $\mathrm{C}$ the decrease of the broad $\Sigma 1$ signal during a further increase of $\Sigma 2$ and CZTSe (101) can be explained by a consumption of nanoparticles in favor of large CZTSe grains.

\subsection{Selenization by fast heating}

The time-resolved data of the slow heating process presented in the previous section gives detailed insights into the reactions taking place during the selenization of $\mathrm{Cu}-\mathrm{Zn}-\mathrm{Sn}-\mathrm{S}$ nanoparticles. However, the process used by Guo et al. ${ }^{1,17}$ uses much higher heating rates. In this section we investigate the selenization with an increased heating rate of $1.7 \mathrm{~K} \mathrm{~s}^{-1}$ and compare the results to the case of the selenization by slow heating $\left(0.05 \mathrm{~K} \mathrm{~s}^{-1}\right)$.

Similar to the slow heating process presented in Fig. 3, in this process a continuous, but faster shift of the $\Sigma 1$ signal from the expected position of CZTS (112) to CZTSe (112) can be seen in Fig. 6b. Also, a weak signal for $\mathrm{Cu}_{2-\delta} \mathrm{Se}(111)$ and a subsequent formation of the $\Sigma 2$ signal directly at the expected position of CZTSe (112) is observed (Fig. 6c and d). However, we point out four important differences compared to the slow heating process: First, no CuSe reflex is detected. Second, the CZTSe (101) reflex forms simultaneous to $\Sigma 2$. This indicates that CZTSe is formed without a prior formation of CTSe or ZnSe. Third, the intensity of $\Sigma 1$ vanishes after about 15 minutes of total annealing time. At the same time, the intensity of $\Sigma 2$ and the minimum crystallite sizes estimated from $\Sigma 2$ reach their maximum values (Fig. 6c and e). Fourth, the cross-section of the final film shows a favorable morphology with a dense layer of large grains (Fig 7). As in the case of slow heating, the EDS mappings of Fig. 7 reveal a correlation of the signals of $\mathrm{Cu}, \mathrm{Zn}, \mathrm{Sn}$, and Se, which is expected for single phased CZTSe. In this sample, no ZnSe was found at the bottom of the large grained layer by TEM-EDS (Fig. S4 $\dagger$ ).

\subsection{Growth model}

The proposed reaction paths for the selenization of $\mathrm{Cu}-\mathrm{Zn}$ Sn-S nanoparticle films by slow and by fast heating are visualized schematically in Fig. 8. Whereas in the case of slow heating, the transformation of the initial $\mathrm{Cu}-\mathrm{Se}$ grains into CZTSe grains is proposed to proceed via intermediately formed CTSe and $\mathrm{ZnSe}$, in the case of fast heating theses intermediate phases seem to be avoided. We note that in the fast heating process the absence of $\mathrm{CuSe}$ prior to $\mathrm{Cu}_{\delta-2} \mathrm{Se}$ could be due to a lower Se partial pressure during the heating ramp compared to the slow heating process. This may be caused by a nonuniform temperature distribution during the fast heating within the reaction box if the temperature of the selenium or the walls of the reaction box does not increase as quickly as the film temperature.

Regarding that CZTS is known to be stable below $500{ }^{\circ} \mathrm{C},{ }^{24}$ the formation of $\mathrm{Cu}-\mathrm{Se}$ at lower temperatures seems surprising. A high chalcogen pressure is expected to stabilize the 

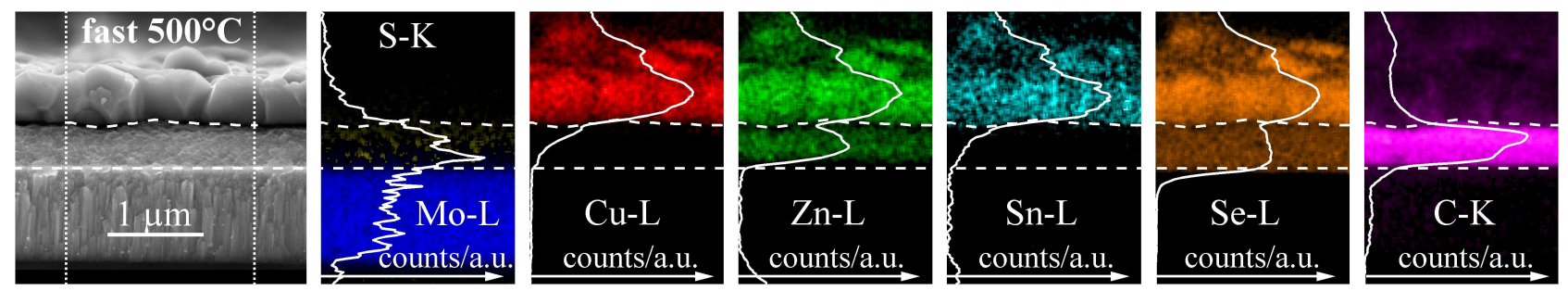

Figure 7 SEM images and EDS mappings of a cross-section of the sample that was selenized by fast heating $\left(1.7 \mathrm{~K} \mathrm{~s}^{-1}\right)$ to $500^{\circ} \mathrm{C}$.

(a)

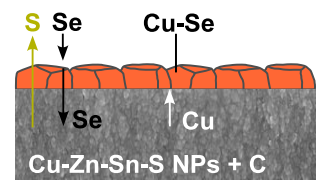

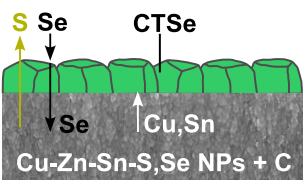

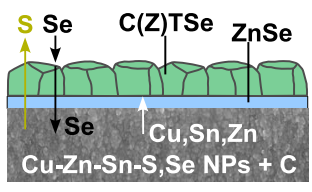

(b)

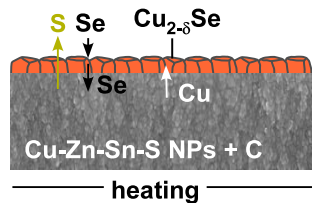

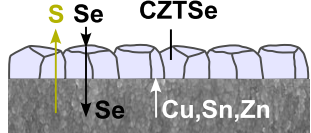

Cu-Zn-Sn-S, Se NPs + C

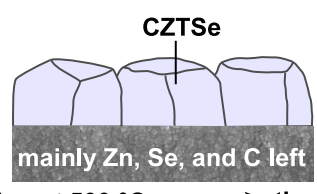

Figure 8 Schematic model of the formation of large grained CZTSe from Cu-Zn-Sn-S nanoparticles by (a) slow heating $\left(0.05 \mathrm{~K} \mathrm{~s}^{-1}\right)$ and (b) fast heating $\left(1.7 \mathrm{~K} \mathrm{~s}^{-1}\right)$ with a subsequent annealing at $500^{\circ} \mathrm{C}$. $\mathrm{Cu}-\mathrm{Se}$ stands for $\mathrm{CuSe}$ or $\mathrm{Cu}_{2-\delta} \mathrm{Se}, \mathrm{CTSe}$ for $\mathrm{Cu}_{2} \mathrm{SnSe} \mathrm{Sn}_{3}, \mathrm{C}(\mathrm{Z}) \mathrm{TSe}$ for a Zn-deficient CZTSe phase, CZTSe for $\mathrm{Cu}_{2} \mathrm{ZnSnSe}_{4}$, and NP for nanoparticles.

kesterite structure according to Scragg et al. ${ }^{25}$ If CZTS does not decompose, the formation of CuSe out of CZTS would not be expected. The observed formation of $\mathrm{Cu}-\mathrm{Se}$ at low temperatures can, however, be explained by the presence of smaller size $\mathrm{Cu}$-rich particles with a low crystallinity in the nanoparticle precursor film, which was found by X-ray absorption spectroscopy and EDS (details will be published elsewhere). In these particles, the bonding of $\mathrm{Cu}$ can be expected to be weaker than in CZTS, enabling an early formation of $\mathrm{Cu}-$ $\mathrm{Se}$. This explanation is further supported by the observation that during the rise of the reflexes of $\mathrm{CuSe}$ and $\mathrm{Cu}_{2-\delta} \mathrm{Se}$, the intensity of the broad $\Sigma 1$ peak attributed to the nanoparticles rises as well (Fig. 3c and d). Even though an increase of the 112 reflex would be expected if S in CZTS is replaced by Se, this cannot account for the full intensity increase. Possible explanations are healing of structural defects and an increase of the crystallinity of the nanoparticles. The fact that the $\Sigma 1$ signal does not decrease, supports the interpretation that the $\mathrm{Cu}$ forming the $\mathrm{Cu}$-Se grains stems from nanoparticles with a low crystallinity, that is from nanoparticles that do not contribute to the diffraction signal. The small radius of the surfaces of the nanoparticles can also be expected to lead to a lower stability of the CZTS lattice, which might shift the decomposition of
CZTS into binaries to lower temperatures. These considerations suggest that a low crystallinity of the nanoparticles supports a fast selenization at low temperatures. The influence of the particles size and crystallinity on the reaction path should be investigated in detail in future studies.

As a consequence of the initial formation of copper selenides at the surface, the subsequent formation of CZTSe starts under $\mathrm{Cu}$-rich conditions. This may have important consequences on the formation of large grains and defects in the CZTSe lattice. It is well known from the literature that an intermediate $\mathrm{Cu}$-rich composition during co-evaporation of $\mathrm{Cu}(\mathrm{In}, \mathrm{Ga}) \mathrm{Se}_{2}$ absorber films is favorable for the formation of a large grained morphology and for high energy conversion efficiencies of resulting solar cells. ${ }^{35,36}$ Also for co-evaporated CZTS it was shown that a $\mathrm{Cu}$-rich growth has a positive effect on the formation of large grains. ${ }^{13,15,37}$ In these cases, the $\mathrm{Cu}$ excess is subsequently removed by etching ${ }^{13}$ or by a $\mathrm{Cu}-$ poor co-evaporation stage ${ }^{15}$ to obtain single phased CZTS. It is noteworthy, that in the processes investigated here, a $\mathrm{Cu}$ rich growth and a subsequent extinction of copper selenide is purely induced by a separation and subsequent mixing of the cations. Moreover, the $\mathrm{Cu}$-rich growth occurs despite an overall $\mathrm{Cu}$-poor composition of the film. That means that the $\mathrm{Cu}$ - 
rich growth and subsequent compensation of the $\mathrm{Cu}$ excess is self-controlled and does not need sophisticated deposition rate control as is needed for the co-evaporation processes.

It is interesting to notice that the rise of the $\Sigma 2$ signal is correlated with the transition from $\mathrm{CuSe}$ to $\mathrm{Cu}_{2-\delta} \mathrm{Se}$ (Fig. $3 \mathrm{c}$ and d). We suggest the following possible reason for this: The Se sublattice of $\mathrm{Cu}_{2-\delta}$ Se has the same cubic closest pack structure as the Se sublattice of CTSe, ZnSe and CZTSe with similar lattice constants, ${ }^{20,38-40}$ whereas CuSe has a hexagonal Se sublattice. ${ }^{27}$ Therefore, the transition from CuSe to CTSe would be necessarily connected with a change of the Se sublattice whereas the transition from $\mathrm{Cu}_{2-\delta}$ Se to CTSe in principle only necessitates the exchange of cations, which could be faster than a change of the sublattice. ${ }^{41}$ As the positions of $\mathrm{Cu}_{2-\delta} \mathrm{Se}(111)$ does not coincide with the position of $\Sigma 2$ (Fig. 2c), a purely topotactical transition from $\mathrm{Cu}_{2-\delta}$ Se to CTSe can be ruled out. In contrast, no separate signals can be seen for CTSe or ZnSe and no peak peak broadening of $\Sigma 2$ occurs between period A and B. From this follows that if CTSe, $\mathrm{ZnSe}$, and CZTSe coexist, they should be lattice-matched. Therefore, the transition from CTSe and ZnSe to CZTSe might take place via a topotactical exchange of the cations within the Se sublattice. ${ }^{42}$

A common problem of the synthesis of absorber films by selenization of nanoparticle films is an accumulation of carbon from the precursor beneath the sintered layer. ${ }^{7,8} \mathrm{~A}$ high density of carbon beneath the CZTSe film can be seen in the EDS mappings in Fig. 7. The carbon accumulation can be explained by the observed cation diffusion out of the initial carbon containing - nanoparticle precursor film to form large grains on top of the film: As the cations diffuse out and the thickness of the nanoparticle layer decreases, the carbon density increases within the remaining nanoparticle layer. The presence of carbon within the nanoparticle layer might prevent the merging of the nanoparticles. If this is the case, the outdiffusion of the cations to the surface of the nanoparticle layer would be the only possibility to form large grains. We note that the density of carbon is largest in the upper part of the nanoparticle layer (Fig. 7). This is expected if the outdiffusion of the cations is largest near the surface of the nanoparticle layer. Guo et al. found that increasing the thickness of the nanoparticle precursor film does not lead to an increasing thickness of the large grained film, but only in an increasing thickness of the remaining nanoparticle layer. ${ }^{7}$ We suggest that an increasing density of carbon in the nanoparticle layer near the interface to the large grained layer may not only block the merging of the nanoparticles, but also inhibit the outdiffusion of the cations, leaving some of the cations trapped in the lower part of the nanoparticle layer. If this is the case, the synthesis of thicker films of large grains from nanoparticles prerequisites a reduction of the carbon content of the precursor film.

\section{Conclusion}

We showed that during the selenization of $\mathrm{Cu}-\mathrm{Zn}-\mathrm{Sn}-\mathrm{S}$ nanoparticle precursor films, large grains of selenides, starting with copper selenide, form on top of the nanoparticle layer as soon as selenium is incorporated into the film. This is accompanied by a slower replacement of S by Se in the nanoparticle layer. We conclude that the large grains are formed by cations that diffuse from the nanoparticle film to the surface to react with selenium from the gas phase and that the initially formed copper selenides are transformed into CZTSe by subsequent incorporation of $\mathrm{Sn}$ and $\mathrm{Zn}$. This reaction path leads to an intermediate partial separation of the cations and - in the case of a slow heating rate - to the intermediate formation of $\mathrm{CuSe}$, $\mathrm{Cu}_{2-\delta} \mathrm{Se}, \mathrm{Cu}_{2} \mathrm{SnSe}_{3}$ and $\mathrm{ZnSe}$. The separation of the cations and the formation of the intermediate phases can be reduced by increasing the heating rate. It is proposed that the observed accumulation of carbon beneath the large grained CZTSe layer is a consequence of the outdiffusion of the cations out of the carbon containing nanoparticle layer.

As a consequence of the formation of copper selenide $\left(\mathrm{Cu}_{2-\delta} \mathrm{Se}\right)$ prior to CZTSe observed in both slow and fast heating, the CZTSe phase initially grows under $\mathrm{Cu}$-rich conditions, which is known to be favorable for the formation of large CZTSe grains. The intermediate $\mathrm{Cu}$-rich growth followed by a subsequent complete consumption of copper selenide is induced by separation and subsequent mixing of the cations and is therefore self-controlled.

\section{Acknowledgment}

Special thanks go to Jakob Lauche, Cornelia Streeck and Guido Wagener for their support during the real-time measurements at BESSY and Stephan Brunken for his help with the SEM and EDS measurements. A special thanks to Charles Hages and Kevin Brew at Purdue for sample preparation. BW would like to thank the NSF Solar Economy IGERT (DGE0833366) for funding. The funding of OZ by the German Ministry of Education and Research within the project PINET (03SF0358F) is gratefully acknowledged.

\section{References}

[1] Q. Guo, G. M. Ford, H. W. Hillhouse and R. Agrawal, Nano Lett., 2009, 9, 3060-3065.

[2] Q. Guo, G. M. Ford, W.-C. Yang, B. C. Walker, E. A. Stach, H. W. Hillhouse and R. Agrawal, J. Am. Chem. Soc., 2010, 132, 17384-17386.

[3] G. M. Ford, Q. Guo, R. Agrawal and H. W. Hillhouse, Chem. Mater., 2011, 23, 2626-2629. 
[4] C. N. Bucherl, K. R. Oleson and H. W. Hillhouse, Current Opinion in Chemical Engineering, 2013.

[5] Q. Guo, G. M. Ford, R. Agrawal and H. W. Hillhouse, Prog. Photovolt: Res. Appl., 2013, 21, 64-71.

[6] S. Jeong, B.-S. Lee, S. Ahn, K. Yoon, Y.-H. Seo, Y. Choi and B.-H. Ryu, Energy Environ. Sci., 2012, 5, 75397542 .

[7] Q. Guo, G. M. Ford, W.-C. Yang, C. J. Hages, H. W. Hillhouse and R. Agrawal, Sol. Energy Mater. Sol. Cells, 2012, 105, $132-136$.

[8] Y. Cao, M. S. Denny, J. V. Caspar, W. E. Farneth, Q. Guo, A. S. Ionkin, L. K. Johnson, M. Lu, I. Malajovich, D. Radu, H. D. Rosenfeld, K. R. Choudhury and W. Wu, J. Am. Chem. Soc., 2012, 134, 15644-15647.

[9] T. K. Todorov, J. Tang, S. Bag, O. Gunawan, T. Gokmen, Y. Zhu and D. B. Mitzi, Adv. Energy Mater., 2013, 3, 3438.

[10] H. Katagiri, K. Jimbo, S. Yamada, T. Kamimura, W. S. Maw, T. Fukano, T. Ito and T. Motohiro, Appl. Phys. Express, 2008, 1, 041201.

[11] V. Chawla and B. Clemens, 35th IEEE Photovoltaic Specialists Conference (PVSC), 2010, pp. 001902-001905.

[12] K. Wang, O. Gunawan, T. Todorov, B. Shin, S. J. Chey, N. A. Bojarczuk, D. Mitzi and S. Guha, Appl. Phys. Lett., 2010, 97, 143508 .

[13] B.-A. Schubert, B. Marsen, S. Cinque, T. Unold, R. Klenk, S. Schorr and H.-W. Schock, Prog. Photovolt: Res. Appl., 2011, 19, 93-96.

[14] A. Redinger, D. M. Berg, P. J. Dale and S. Siebentritt, J. Am. Chem. Soc., 2011, 133, 3320-3323.

[15] I. Repins, C. Beall, N. Vora, C. DeHart, D. Kuciauskas, P. Dippo, B. To, J. Mann, W.-C. Hsu, A. Goodrich and R. Noufi, Sol. Energy Mater. Sol. Cells, 2012, 101, 154 -159 .

[16] A. Moholkar, S. Shinde, A. Babar, K.-U. Sim, H. K. Lee, K. Rajpure, P. Patil, C. Bhosale and J. Kim, J. Alloys Compd., 2011, 509, 7439 - 7446.

[17] Q. Guo, H. W. Hillhouse and R. Agrawal, J. Am. Chem. Soc., 2009, 131, 11672-11673.

[18] H. Rodriguez-Alvarez, I. Kötschau and H. Schock, J. Cryst. Growth, 2008, 310, 3638 - 3644.
[19] C. Genzel, I. Denks, J. Gibmeier, M. Klaus and G. Wagener, Nucl. Instr. Meth. Phys. Res. A, 2007, 578, 23 33.

[20] H. McMurdie, M. Morris, E. Evans, B. Paretzkin, W. Wong-Ng, Y. Zhang and C. Hubbard, Powder Diffr., $1986,1,334-345$.

[21] B. B. Sharma, R. Ayyar and H. Shing, phys. stat. sol. (a), 1977, 40, 691-697.

[22] G. E. Delgado, A. J. Mora, G. Marcano and C. Rincon, Mater. Res. Bull., 2003, 38, 1949-1955.

[23] R. Mainz and R. Klenk, J. Appl. Phys., 2011, 109, 123515 .

[24] A. Weber, R. Mainz and H. W. Schock, J. Appl. Phys., 2010, 107, 013516.

[25] J. J. Scragg, T. Ericson, T. Kubart, M. Edoff and C. Platzer-Björkman, Chem. Mater., 2011, 23, 46254633.

[26] J. I. Langford and A. J. C. Wilson, J. Appl. Crystallogr, 1978, 11, 102-113.

[27] S. Stolen, H. Fjellvag, F. Gronvold, J. Sipowska and E. Westrum Jr., J. Chem. Thermodyn., 1996, 28, 753766.

[28] N. Bikkulova, S. Danilkin, H. Fuess, E. Yadrovskii, A. Beskrovnyi, A. Skomorokhov, Z. Yagafarova and G. Asylguzhina, Crystallography Reports, 2003, 48, 370-373.

[29] D. Chakrabarti and D. Laughlin, Bull. Alloy Phase Diagrams, 1981, 2, 305-315.

[30] D. Apel, M. Klaus, C. Genzel and D. Balzar, Z. Kristallogr., 2011, 226, 934-943.

[31] I. M. Kötschau and H. W. Schock, J. Appl. Crystallogr., 2006, 39, 683-696.

[32] J. Just, D. Lutzenkirchen-Hecht, R. Frahm, S. Schorr and T. Unold, Appl. Phys. Lett., 2011, 99, 262105.

[33] I. Olekseyuk, L. Gulay, I. Dydchak, L. Piskach, O. Parasyuk and O. Marchuk, Journal of Alloys and Compounds, 2002, 340, 141 - 145.

[34] S. Schorr, H.-J. Hoebler and M. Tovar, Eur. J. Mineral., 2007, 19, 65-73.

[35] R. Caballero, C. Kaufmann, V. Efimova, T. Rissom, V. Hoffmann and H. Schock, Prog. Photovolt: Res. Appl., 2013, 21, 30-46. 
[36] S. Niki, M. Contreras, I. Repins, M. Powalla, K. Kushiya, S. Ishizuka and K. Matsubara, Prog. Photovolt: Res. Appl., 2010, 18, 453-466.

[37] I. Repins, N. Vora, C. Beall, S.-H. Wei, Y. Yan, M. Romero, G. Teeter, H. Du, B. To, M. Young and R. Noufi, MRS Proceedings, 2011, 1324, year.

[38] T. Wada, N. Kohara, T. Negami and M. Nishitani, J. Mater. Res., 1997, 12, 1456-1462.

[39] L. D. Gulay, M. Daszkiewicz, T. A. Ostapyuk, O. S. Klymovych and O. F. Zmiy, Acta Crystallogr., 2010, C66, i58-i60.

[40] S. Schorr, Thin Solid Films, 2007, 515, 5985 - 5991.

[41] F. Hergert and R. Hock, Thin Solid Films, 2007, 515, $5953-5956$.

[42] A. Weber, S. Schmidt, D. Abou-Ras, P. SchubertBischoff, I. Denks, R. Mainz and H. W. Schock, Appl. Phys. Lett., 2009, 95, 041904. 